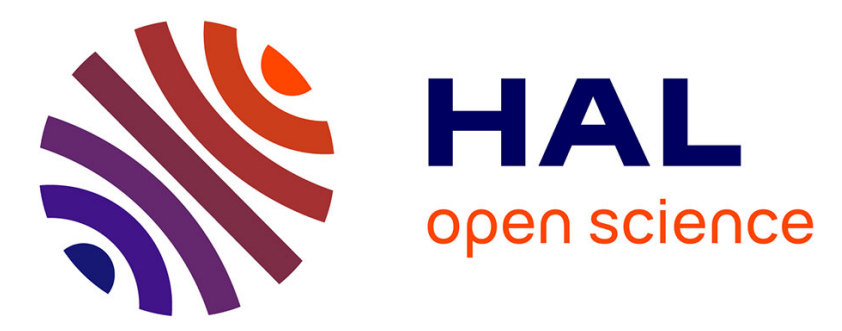

\title{
A New Analysis of the Microstructure of Ni-Based Single-Crystal Superalloys: Relevant Topological Parameters for Efficient Microstructural Modeling
}

\author{
M. Degeiter, M. Perrut, B. Appolaire, Y. Le Bouar, A. Finel
}

\section{> To cite this version:}

M. Degeiter, M. Perrut, B. Appolaire, Y. Le Bouar, A. Finel. A New Analysis of the Microstructure of Ni-Based Single-Crystal Superalloys: Relevant Topological Parameters for Efficient Microstructural Modeling. Superalloys 2016, Sep 2016, SEVEN SPRINGS, United States. 10.1002/9781119075646.ch35 . hal-01404452

\section{HAL Id: hal-01404452 https://hal.science/hal-01404452}

Submitted on 28 Nov 2016

HAL is a multi-disciplinary open access archive for the deposit and dissemination of scientific research documents, whether they are published or not. The documents may come from teaching and research institutions in France or abroad, or from public or private research centers.
L'archive ouverte pluridisciplinaire HAL, est destinée au dépôt et à la diffusion de documents scientifiques de niveau recherche, publiés ou non, émanant des établissements d'enseignement et de recherche français ou étrangers, des laboratoires publics ou privés. 


\title{
A NEW ANALYSIS OF THE MICROSTRUCTURE OF NI-BASED SINGLE-CRYSTAL SUPERALLOYS : RELEVANT TOPOLOGICAL PARAMETERS FOR EFFICIENT MICROSTRUCTURAL MODELING
}

\author{
M. Degeiter ${ }^{1}$, M. Perrut ${ }^{1}$, B. Appolaire ${ }^{2}$, Y. Le Bouar ${ }^{2}$, A. Finel $^{2}$ \\ ${ }^{1}$ DMSM, Onera, 29 avenue de la Division Leclerc, F-92322 Châtillon, France \\ ${ }^{2}$ LEM, CNRS-Onera, 29 avenue de la Division Leclerc, F-92322 Châtillon, France
}

Keywords: blade superalloy, $\gamma^{\prime}$ precipitation, elasticity, modeling, pattern formation, phase diffusion

\begin{abstract}
Two topological parameters derived from pattern formation theory are introduced, and their potential to accurately describe the elasticity-induced coarsening in the microstructure modeling of Ni-based singlecrystal superalloys at macroscale in $2 \mathrm{D}$ is investigated. For that purpose, and given the primordial role of the spatial arrangement of the precipitates on microstructure evolution, two-dimensional phasefield calculations of ideal microstructures undergoing slight topological defects have been carried out. The unstable and stable responses of the system respectively against Eckhaus and zigzag instabilites is discussed and quantitatively described by means of the developed topological parameters $\psi_{x}$ and $\psi_{y}$.
\end{abstract}

\section{Introduction}

High pressure turbine (HPT) blades possess outstanding mechanical properties at high temperature, which stem from the microstructure of the singlecrystal nickel-base superalloy in which they are designed. This two-phase microstructure consists of cuboidal precipitates of the $\mathrm{L} 1_{2}$-ordered phase $\gamma^{\prime}$, coherently embedded in the disordered matrix phase $\gamma$, and globally aligned along its cubic directions. Considering the high volume fraction of $\gamma^{\prime}$-phase in these alloys (68\% at room temperature for AM1), the microstructure evolves into a cubic pattern of cuboidal precipitates, only separated by narrow channels of matrix phase.

During gas turbine operation, the size, morphology and spatial arrangement of $\gamma^{\prime}$-precipitates continuously evolve due to the thermomechanical loading seen by the blade, which impacts its mechanical properties in return. In order to improve the service life of HPT blades, and thus enhance the stability of the microstructure, there is still a need for efficient microstructural modeling at the scale of the blade.
Single-crystal superalloys are prone to complex microstructure evolution, due to the effects of elasticity on precipitation kinetics. Without external loading, elasticity arises from the $\gamma / \gamma^{\prime}$ misfit, inducing coherency strains at the precipitate-matrix interfaces, and thus long-range interacting elastic fields in the alloy [1-5]. During coarsening, diffusion of the alloying elements is strongly influenced by elasticity, leading to complex evolutions such as morphological changes [6-10] and precipitate alignment [11-14]. Though the involved mechanisms are well-known, the main difficulty lies in their coupling. The latter renders the understanding and modeling of this microstructure a difficult task, especially when designing at macroscale, at which precipitation is usually modeled without elastic effects [15].

The coarsening kinetics in two phase solids with elastic effects has successfully been modeled at mesoscale through sharp [16-18] and diffuse $[19,20]$ interface approaches. The sharp interface approach is very efficient at modeling coarsening with large numbers of precipitates, and provides an exact description of the interface motion. However, it is not suitable for the modeling of high volume fraction alloys, since it does not allow for topological singularities such as precipitates merging. The phase field, or diffuse interface approach, describes interfaces as steep variations of a continuous field. This theory allows one to catch complex topological evolution during coarsening, even at high volume fractions, though the requirement of a very fine mesh prevents the modeling of large numbers of precipitates.

Given the prominence of the spatial arrangement of precipitates on microstructure evolution in these alloys, our aim is to develop a simplified phenomenological model, based on a relevant topological parameter, to account for the influence of elasticity 
on coarsening at macroscale.

Previous studies have been carried out so as to quantitatively describe both morphological and topological changes during microstructure evolution. A number of shape factors has been developed in the literature, among which the $a_{2}^{R}$ coefficient has been used to obtain the bifurcation diagram of an isolated precipitate with a dilatational misfit in $2 \mathrm{D}$ [9]. The energy-minimizing shape has been shown to evolve from four-fold to two-fold symmetry for a specific value of the elastic-to-interfacial energy ratio. It was later used to assess the influence of elastic interactions on precipitate morphology by comparing the dispersion of its value with respect to that of the isolated particle [17]. 2D and 3D moment invariants have also been used as a means to follow the morphological transition which the precipitates undergo during rafting [21]. The authors were able to define the onset of rafting in microstructures with both negative and positive lattice misfits. Regarding the description of spatial arrangements, power spectra have mostly been used to qualitatively describe the development of spatial correlations, and were obtained by the Fourier transformation of the microstructure itself $[17,22]$. Radial distribution and pair correlation functions were able to provide a more quantitative description of precipitate alignment [17]. The authors showed that the microstructure tends to evolve towards a unique configuration, regardless of the initial arrangement of precipitates, the only governing factor being the elastic-to-interfacial energy ratio.

To our knowledge, none of these topological parameters has ever been used to predict microstructural evolution. Their use has been restricted to the description of spatial correlations, since they do not seem relevant to the building of evolution laws.

The purpose of the present work is to introduce new topological parameters, derived from pattern formation theory, and evaluate their potential to account for elastic effects in the modeling of microstructure evolution. We use phase field calculations as virtual experiments of microstructures undergoing an isothermal heat treatment in the absence of mechanical loading. The initial conditions are set in simple topological situations which are then easier to analyze.

\section{Topological parameters}

Pattern formation theory focuses on systems which evolve from spatially uniform to patterned structures when driven away from equilibrium [23]. Geometrical patterns emerge as a result of complex nonlinear evolution, and may be analyzed regardless of the phenomenons or of the nature of the system (e.g. Rayleigh-Bénard convection rolls [24], Faraday waves, solidification fronts, etc.).

The general idea behind pattern formation is to prescind from what the authors in [23] call "microscopic" considerations (i.e. the elementary mechanisms involved) so as to investigate dynamical systems from a more general standpoint. Within this scope, this field provides theoretical tools for the phenomenological description of spatially modulated structures.

With the ambition to apply this framework to the study of microstructure evolution, we define a reference state for a $2 \mathrm{D} \gamma / \gamma^{\prime}$ microstructure as a perfectly periodic square network of identical precipitates. We assume the following form for the Al concentration field :

$$
\begin{aligned}
c_{a l} & =c_{a l}^{0}+\frac{\Delta c}{2}\left(\cos \left(k x+\psi_{x}\right)+\cos \left(k y+\psi_{y}\right)\right) \\
& =c_{a l}^{0}+f_{x}(x, y, t)+f_{y}(x, y, t)
\end{aligned}
$$

where $k$ is a constant wave number, and $\lambda=2 \pi / k$ is the wavelength of the modulated square structure $\left(\lambda_{x}=\lambda_{y}=\lambda\right) . \psi_{x}$ and $\psi_{y}$ are phases, which respectively represent horizontal and vertical deviations from the reference positions in the square lattice, and are either of a topological or a morphological nature. $\psi_{x}$ and $\psi_{y}$ are the relevant parameters to be extracted, insofar as they provide valuable information on the spatial arrangement of precipitates.

We calculate $\psi_{x}$ and $\psi_{y}$ from the image of a microstructure obtained by M. Cottura et al. through phase field modeling [20], as shown in Figure 1. Since phase values range from 0 to $2 \pi$ (0 and $2 \pi$ being the same phase value), $\psi_{x}$ and $\psi_{y}$ phase maps in Figure 1 are represented in periodic gray-scale for easier readability. $\psi_{x}$ (resp. $\psi_{y}$ ) is spatially uniform for horizontally (resp. vertically) regular patterns, and varies as the horizontal (resp. vertical) regularity is lost near topological defects. Thus, topological and morphological imperfections in the precipitate arrangement are highlighted by phase 

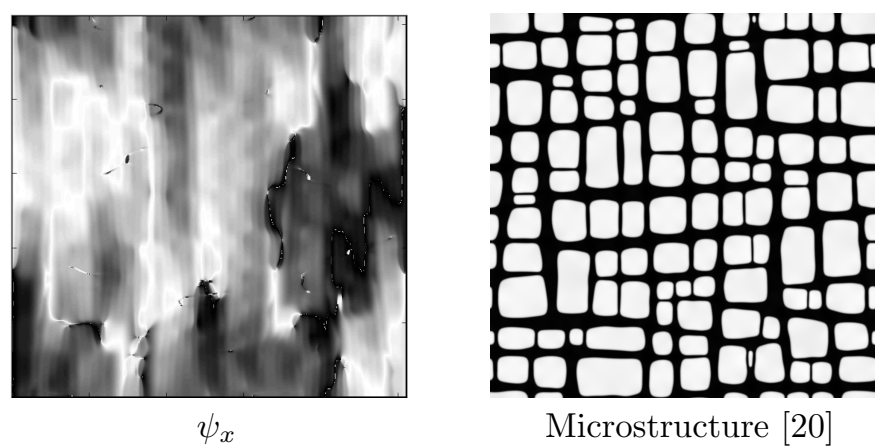

Microstructure [20]

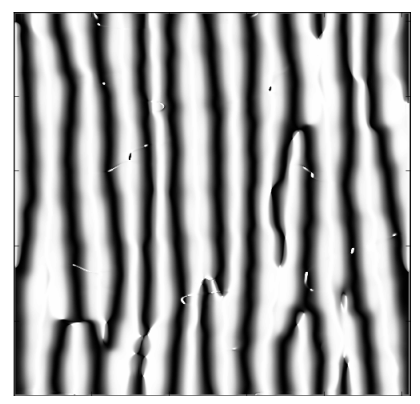

$f_{x}$

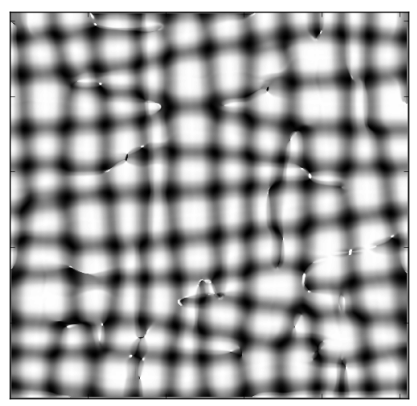

$f_{x}+f_{y}$

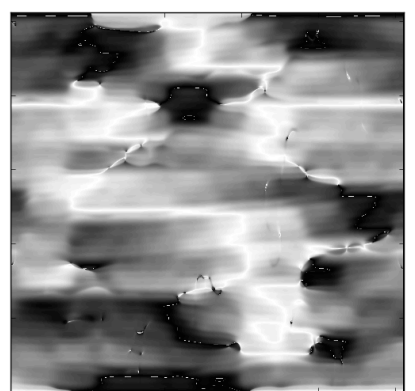

$\psi_{y}$

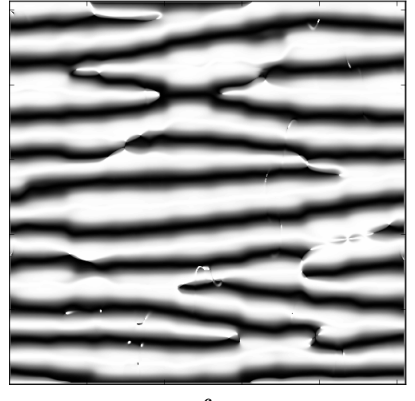

$f_{y}$

Figure 1: Phase-based approach of microstructural analysis : example of calculated phases and associated 1D-periodic functions of a microstructure.

variations.

With the knowledge of $k, \psi_{x}$ and $\psi_{y}$, the concentration field can be decomposed into two contributions $f_{x}$ and $f_{y}$, which physically correspond to its horizontal and vertical modulations. These 1D-periodic functions make the quantitative analysis of period modulations, channel branches or terminations an easier task. Since most of the topological defects are "forks" or "dislocations" in the pattern (as clearly visible on $f_{x}$ and $f_{y}$ ), the question as to why they are present in the microstructure will be discussed in the following sections. Eventually, the microstructure can be reconstructed simply by adding $f_{x}$ and $f_{y}$.

Even if $\psi_{x}$ and $\psi_{y}$ have been shown to hold great potential with regards to the topological description of $2 \mathrm{D}$ microstructures, the question of their evolution through time still needs to be addressed in order to build a phase-based model for microstructure evolution. In pattern formation, phase evolution is commonly described by a diffusion equation [23]. The phase equation, or phase diffusion equation, was first introduced by Pomeau and Manneville to phenomenologically investigate the consequences of long-wavelength distortions on a spatially periodic convective flow [25]. By analogy, we postulate the following low-order diffusion equations for $\psi_{x}$ and $\psi_{y}$ :

$$
\begin{aligned}
& \frac{\partial \psi_{x}}{\partial t}=D_{x x} \frac{\partial^{2} \psi_{x}}{\partial x^{2}}+D_{x y} \frac{\partial^{2} \psi_{x}}{\partial y^{2}} \\
& \frac{\partial \psi_{y}}{\partial t}=D_{y x} \frac{\partial^{2} \psi_{y}}{\partial x^{2}}+D_{y y} \frac{\partial^{2} \psi_{y}}{\partial y^{2}}
\end{aligned}
$$

where $D_{x x}$ and $D_{x y}$ (resp. $D_{y x}$ and $D_{y y}$ ) are the phase diffusion coefficents associated to $\psi_{x}$ (resp. $\left.\psi_{y}\right)$, and depend on the physical parameters of the system. Their signs govern the stability of the pattern respectively against horizontal and vertical perturbations of $\psi_{x}$ and $\psi_{y}$. If the system is unstable (i.e. negative phase diffusion coefficient) against a specific phase perturbation, higher-order terms must be added in the phase equations to properly control the system evolution [23].

This formalism has recently been used in the case of 1D-periodic patterns, to investigate the stability of lamellar eutectic growth [26].

The variations of $\psi_{x}$ and $\psi_{y}$ are slow in space and time, as compared to that of the concentration field. Therefore, a phase-based calculation would require 
a smaller number of meshing points, possibly allowing the transition of microstructural modeling to macroscale. Investigating the relevance of the phase equations (2) to the modeling of microstructure evolution in Ni-based single-crystal superalloys is a work still in progress.

\section{Phase field model}

To better understand how elasticity affects coarsening kinetics through the spatial arrangement of precipitates, we conduct a perturbative analysis on the $2 \mathrm{D}$ reference state defined above, by using phase field calculations as virtual experiments.

The superalloy is modeled as an effective binary alloy, and the local concentration field $c(\underline{\boldsymbol{r}}, t)$ ensures the description of the microstructure. The four translational variants of the $\gamma^{\prime}$ phase, usually described by three non-conservative structural fields, have not been implemented. Consequently, the ordered crystal structure of the precipitates is not taken into account in our calculations, restricting their relevance to the instant preceding coalescence.

The mesoscopic free energy functional $F$ is decomposed into a chemical $F_{c h}$ and an elastic $F_{e l}$ contribution :

$$
F=F_{c h}(c)+F_{e l}\left(c, \underline{\underline{\varepsilon}}^{e l}\right)
$$

where $\underline{\varepsilon}^{e l}$ is the elastic strain tensor. The evolution of the conserved concentration field with time is governed by the Cahn-Hilliard equation which, assuming a linear constitutive relationship, is written :

$$
\frac{\partial c}{\partial t}(\underline{\boldsymbol{r}}, t)=M \nabla^{2} \frac{\delta F}{\delta c(\underline{\boldsymbol{r}}, t)}
$$

where the kinetic coefficient $M$ is such as to recover the interdiffusion coefficent $D_{A l / N i}$ in $\gamma$, and assumed constant. The chemical free energy of the system is written as :

$$
F_{c h}(c)=\int_{V}\left(f_{h o m}(c)+\frac{\alpha}{2}|\nabla c|^{2}\right) d V
$$

where $V$ is the volume, $f_{\text {hom }}$ is the homogeneous free energy density and the gradient term accounts for the energy cost of the interfaces. $\alpha$ is the gradient energy coefficient related to the value of the interface energy, which was chosen isotropic. Since the value of the non-dimensional gradient energy coefficient $\tilde{\alpha}$ governs the discretization of the diffuse interfaces, $\alpha$ was chosen to ascertain their sufficient width.
The homogeneous free energy density $f_{\text {hom }}$ is taken as a conventional double-well function of the following form :

$$
f_{\text {hom }}(c)=4\left[-\frac{1}{2}\left(c-c_{m}\right)^{2}+\frac{\left(c-c_{m}\right)^{4}}{\left(c_{\gamma^{\prime}}^{0}-c_{\gamma}^{0}\right)^{2}}\right]
$$

where $c$ is the concentration field, $c_{\gamma}^{0}=0.15$ and $c_{\gamma^{\prime}}^{0}=0.231$ are the equilibrium concentrations identified on the $\mathrm{Ni}-\mathrm{Al}$ phase diagram at $950^{\circ} \mathrm{C}$, and $c_{m}=\left(c_{\gamma^{\prime}}^{0}+c_{\gamma}^{0}\right) / 2$.

In the framework of linear elasticity, and assuming small strains, the elastic energy is written :

$$
F_{e l}\left(c, \underline{\underline{\varepsilon}}^{e l}\right)=\frac{1}{2} \int_{V} \mathbb{C}(c): \underline{\underline{\varepsilon}}^{e l}: \underline{\underline{\varepsilon}}^{e l} d V
$$

where $\mathbb{C}$ is the local elastic tensor, assumed to linearly depend on $c(\underline{\boldsymbol{r}})$. The local elastic strain $\underline{\boldsymbol{\varepsilon}}^{e l}(\underline{\boldsymbol{r}})$ can be decomposed into :

$$
\underline{\underline{\varepsilon}}^{e l}(\underline{\boldsymbol{r}})=\underline{\underline{\varepsilon}}(\underline{\boldsymbol{r}})-\underline{\underline{\varepsilon}}^{0}(\underline{\boldsymbol{r}})
$$

where $\underline{\underline{\varepsilon}}(\underline{\boldsymbol{r}})$ is the total strain, and $\underline{\underline{\varepsilon}}^{0}(\underline{\boldsymbol{r}})$ is the eigenstrain which is related to the misfit $\delta=2\left(a_{\gamma^{\prime}}-a_{\gamma}\right) /\left(a_{\gamma^{\prime}}+a_{\gamma}\right)$, and thus accounts for the lattice parameter change during the $\gamma \rightarrow \gamma^{\prime}$ transformation. Moreover, the elastic constants of $\gamma^{\prime}$ were chosen to provide a specific value of elastic inhomogeneity in the coherent mixture $\left(\Delta C^{\prime}=50 \%\right)$ [20]. Further details on the computation of the mechanical equilibrium are available at [20,27].

With the aim of modeling an alloy close to AM1, we set the volume fraction of precipitates in our calculations at $70 \%$. This choice compelled us to implement a very fine mesh so as to avoid spurious coalescence, which might have resulted from poorly descretized matrix channels with respect to interface width.

To conduct perturbative analysis on a microstructure during coarsening, we considered the reference state as the starting point of our calculations, thus ignoring the nucleation and growth stages. This was achieved by modeling perfectly periodic arrays of precipitates, whose shapes were set to be four-fold in order to approach their equilibrium morphology. This "relaxation" stage lasted until the energy levels in the system were stabilized, at which time these ideal microstructures had remained stable and perfectly regular. 
We then proceeded to drive the system away from equilibrium by applying slight topological defects in the precipitate arrangements, and monitored the elasticity-driven evolution. We focused on two specific topological perturbations, namely the Eckhaus and zigzag instabilities.

By means of combined attractive and repulsive elastic interactions, we expected the simulated topological perturbations to relax over time, and the microstructure to stabilize itself in order to evolve back towards the reference arrangement.

\section{Results and Discussions}

In this section, the consequences of Eckhaus and zigzag instabilities on the stability of a micrsotructure during isothermal heat treatment at $T=950^{\circ} \mathrm{C}$ and without external loading are investigated. In view of our hypothesis, the evolution of the microstructure is followed until the instant preceding coalescence, and compared to the variation of $\psi_{x}$ and $\psi_{y}$.

For both topological defects, the size of the simulations is $9.6 \times 0.6 \mu \mathrm{m}^{2}$, descretized with $2048 \times 128$ nodes (grid spacing $d=4.7 \mathrm{~nm}$ ).

If the phase maps in Figure 1 are in periodic grayscale for clearer representation, the following phase maps are designed in regular gray-scale. Therefore, extreme phase values $\left(0\right.$ and $2 \pi$ for $\psi_{i=x, y} \in[0 ; 2 \pi]$, $-\pi$ and $\pi$ for $\left.\psi_{i=x, y} \in[-\pi ; \pi]\right)$ will respectively appear black and white, though representing the same phase value.

\section{Eckhaus instability}

An Eckhaus instability corresponds to a horizontal modulation of the precipitate positions by a sine, resulting in axial variations of the precipitate spacing $\lambda_{x}$, and thus of the $\gamma^{\prime}$ volume fraction. The Eckhaus instability applied on the reference microstructure at the initial time is written as the following phase perturbation :

$$
\psi_{x}(x, t=0)=A_{E} \cos \left(K_{E}\left(x-x^{0}\right)\right)
$$

where the dimensionless amplitude $A_{E}=0.47$, and the wave number $K_{E}=2 \pi / \Lambda_{E}$ was taken so that the wavelength $\Lambda_{E}=9.6 \mu \mathrm{m}$. The Eckhaus amplitude $A_{E}$ was chosen with great care in order to avoid overlapping of the diffuse interfaces of neighboring precipitates, which could have led to spurious coalescence.

The resulting evolution of the reference microstructure is presented in Figure 2, and compared to that of $\psi_{x}$ and $\psi_{y}$.

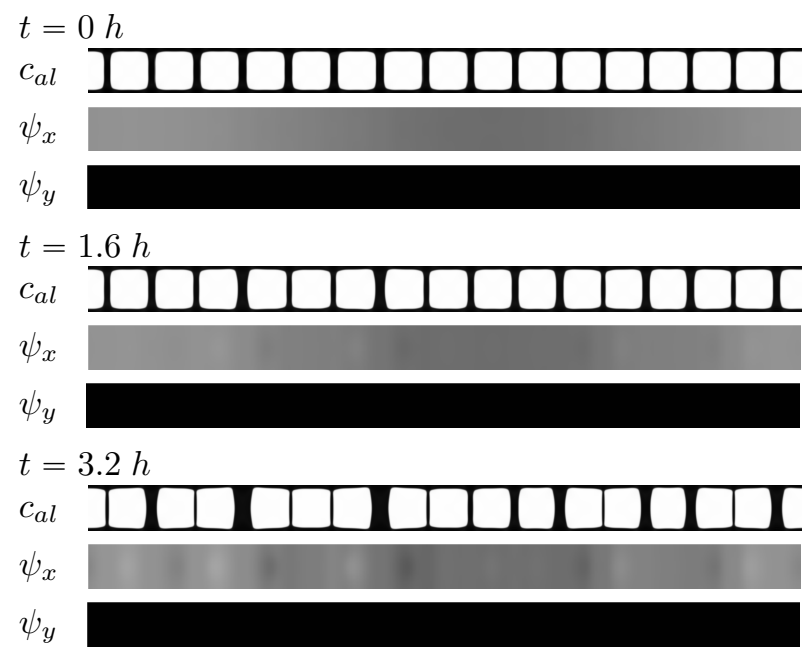

Figure 2: Evolution with time of the Eckhaus instability imposed on an ideal microstructure and associated $\psi_{x}$ and $\psi_{y}$ maps.

At $t=0 h$, the very slight variation in precipitate spacing $\lambda_{x}$ resulting from the Eckhaus instability is difficult to visualize on the microstructure alone, but it can be followed more easily as a modulation of $\psi_{x}$ of moderate amplitude along $\mathrm{x}$ axis. $\psi_{y}$ is uniform throughout space at this instant. The variations of $\lambda_{x}$ amplify with time, and the precipitates gather in groups of two and three. Simulatneously, additional fluctuations of $\psi_{x}$ gradually emerge along $\mathrm{x}$ axis, and $\psi_{y}$ remains steady during the simulation.

As clearly visible in Figure 2, the microstructure is unstable against the Eckhaus instability. Contrary to what was initially expected, a horizontal modulation of precipitate positions does not relax over time. Rather, the Eckhaus instability amplifies, disturbing the regularity of the arrangement further until precipitates coalesce. This progressive loss of horizontal regularity of the pattern is traced by additional fluctuations of $\psi_{x}$, locally arising along $\mathrm{x}$ axis. The vertical regularity of the arrangement is conserved during the simulation, and thus $\psi_{y}$ remains uniform in space through time. 


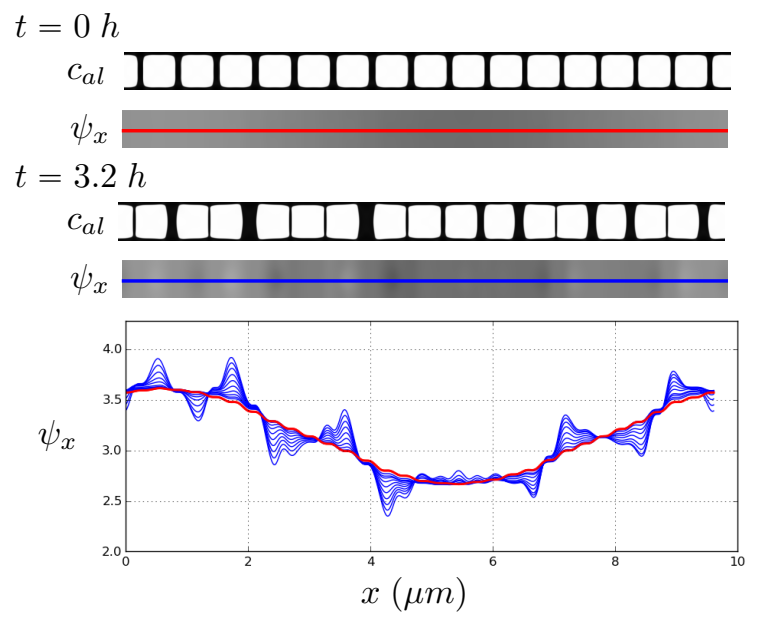

Figure 3: Profile of $\psi_{x}$ along $\mathrm{x}$ : amplification of the Eckhaus instability over $3.2 \mathrm{~h}$.

The use of $\psi_{x}$ as a means to witness the amplification of the Eckhaus instability is completed by the study of its profile over time, as shown in Figure 3. The red curve corresponds to the initial profile of $\psi_{x}$ along $\mathrm{x}$, while the blue curves are the same profile but taken at subsequent times.

The long wavelength modulation of $\psi_{x}$ along $\mathrm{x}$ is centered around the mean phase value, which corresponds to that of the undisturbed pattern. This moderate variation ranges over the entire simulated microstructure, and is consistent with the definition of the Eckhaus instability. The amplification of this instability is traced by additional modulations of $\psi_{x}$, whose growth over the already disturbed topology accelerates with time.

The initial phase modulation owing to the Eckhaus instability is conserved until the last instant, and the developing fluctuations highlight the existence of pattern-destabilizing phase modes, which activate with time. In this situation, the following form for $\psi_{x}$ may be assumed :

$$
\psi_{x}(x, t)=\sum_{i=1}^{N} A_{i}\left(K_{i}\right) e^{\Omega_{i} t} \cos \left(K_{i}\left(x-x_{i}^{0}\right)\right)
$$

where $A_{i}, \Omega_{i}$ and $K_{i}$ are the amplitude, growth rate and wave number of the $i^{\text {th }}$ phase mode. The expression of $\psi_{x}$ in Eq. (10) unveils the involvement of various wavelengths in phase evolution and may allow for the definition of phase modes related to the coalescence of precipitates. The modes which are most threatening for the reference microstructure are expected to be those of lowest wavelength and highest growth rate, since they lead to local and fast fluctuations in the regularity of the pattern.

Eventually, the precipitates which had gathered coalesce, leaving the microstructure as an irregular structure of two-fold-shaped precipitates, and bringing forward the question of a potential stabilization of the pattern at a new $\lambda_{x}^{\prime}>\lambda_{x}$. Considering the postulated diffusion equation (2) for the temporal evolution of $\psi_{x}$ and $\psi_{y}$, we may assert that $D_{x x}<0$ (and by symmetry, $D_{y y}<0$ ) since the system is unstable against Eckhaus-type distortions of its pattern, revealing the probable need for higher-order phase diffusion equations to appropriately describe phase evolution [23].

Eckhaus instabilities have been reported to be responsible for the existence of topological defects in a number of modulated systems [23,28,29], be it for their creation, drift or annihilation. In convectionroll patterns, forks and dislocations in the structure are a direct result of Eckhaus instabilities, which brings forward the question of their involvement in the presence of those visible in $f_{x}$ and $f_{y}$ in Figure 1.

\section{Zigzag instability}

A zigzag instability consists in a transverse modulation of the precipitate positions by a sine, which leads to a wavy configuration of the microstructure. The zigzag instability initially applied to the reference state is written as the following phase perturbation :

$$
\psi_{y}(x, t=0)=A_{Z Z} \cos \left(K_{Z Z}\left(x-x^{0}\right)\right)
$$

where the dimensionless amplitude $A_{Z Z}=1.5$, and the wave number $K_{Z Z}=2 \pi / \Lambda_{Z Z}$ is such that the wavelength $\Lambda_{Z Z}=9.6 \mu \mathrm{m}$ in our simulations. The evolution of the microstructure subjected to this topological defect is presented in Figure 4, along with the corresponding phase maps.

At $t=0 h$, the applied zigzag instability causes the vertical regularity of the microstructure to strongly vary along $\mathrm{x}$ axis. At subsequent time, the wavy configurations of precipitates has decreased in amplitude, and the zigzag is almost completely realigned, proving the perfect alignment of the precipitates to be an energetically-favorable configuration of the microstructure in the first instance. At the final instant, the relaxation of this instability is complete and the horizontal periodicity of the 


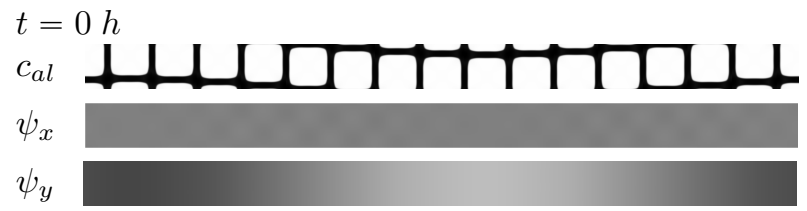

$t=2.5 h$

$c_{a l}$

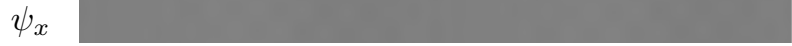

$\psi_{y}$

$t=3.8 h$

$c_{a l}$ मयमापातम

$\psi_{x}$

$\psi_{y}$

Figure 4: Evolution with time of the zigzag instability imposed on an ideal microstructure and associated $\psi_{x}$ and $\psi_{y}$ maps.

square structure has begun to fluctuate along $\mathrm{x}$. Simultaneously, while initially modulated along $\mathrm{x}$, $\psi_{y}$ is almost uniform in space at $t=3.8 h$ and horizontal variations in $\psi_{x}$ arise, though it was uniform at the early stages of the simulation.

The elasticity-driven realignment of the instability proves that the microstructure is stable against zigzag-type distortions of its regularity. Fluctuations in $\lambda_{x}$ appear once the vertical periodicity is found again, highlighting the development of an Eckhaus instability subsequent to the zigzag relaxation. The combined use of $\psi_{x}$ and $\psi_{y}$ allows for a precise tracking of the topological evolution of the microstructure undergoing this defect in the arrangement of its precipitates.

Since the precipitates alignment is traced by the temporal variations of $\psi_{y}$ along $\mathrm{x}$, we study the evolution of its profile over time in Figure 5, prior to the development of the Eckhaus instability (which could also have been followed by analyzing $\psi_{x}$ along $\mathrm{x}$ ). The red and blue curves respectively correspond to the initial profile of $\psi_{y}$ along $\mathrm{x}$, and to the same profile at subsequent times.

In Figure 5, the zigzag relaxation is traced by the decreasing amplitude of the phase modulation, whose decay decelerates over time. Contrary to what was observed for the Eckhaus instability, this result reveals the zigzag phase mode to be patternstabilizing, since its decay corresponds to the

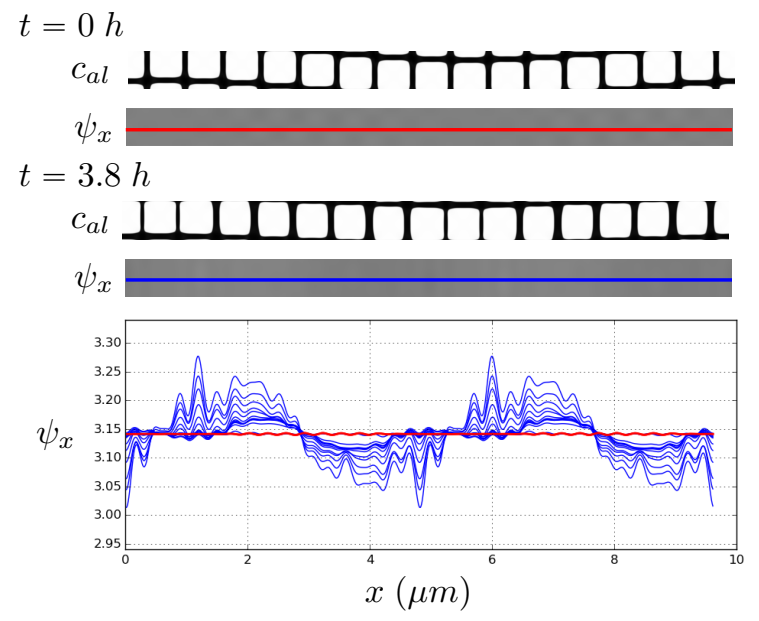

Figure 5: Profile of $\psi_{x}$ along $\mathrm{x}$ : growth of the Eckhaus instability over $3.8 \mathrm{~h}$.

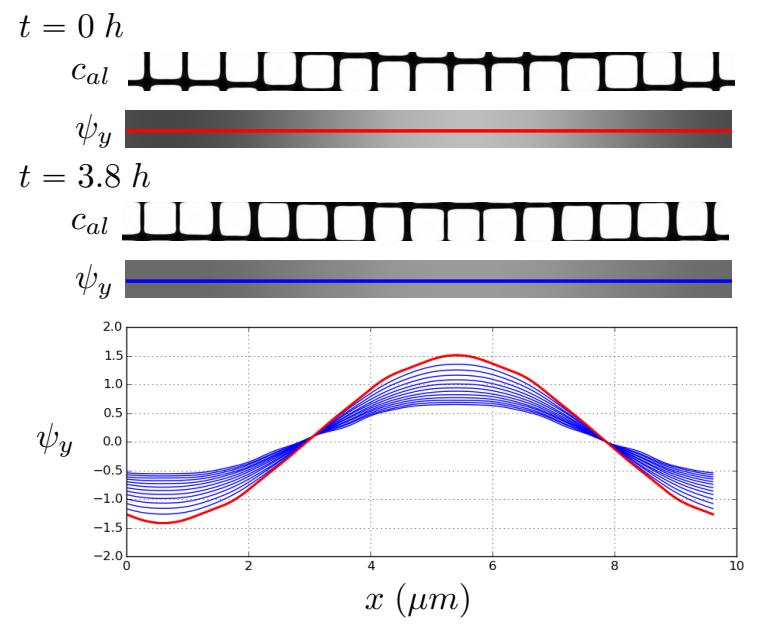

Figure 6: Profile of $\psi_{y}$ along x : relaxation of the zigzag instability over $3.8 \mathrm{~h}$.

evolution of the wavy microstructure back towards the regular state. We write $\psi_{y}$ in the following form :

$$
\psi_{y}(x, t)=A_{Z Z} e^{\Omega_{Z Z} t} \cos \left(K_{Z Z}\left(x-x^{0}\right)\right)
$$

where the amplitude $A_{Z Z}$ and wave number $K_{Z Z}$ are known, and $\Omega_{Z Z}$ is the decay rate.

We show in Figure 6 the evolution of the zigzag amplitude $A_{Z Z}$ with time, and the fitted data to a decreasing exponential, from which we are able to extract the decay rate $\Omega_{Z Z}=-1 / \tau_{Z Z}$.

Substituting Eq. (12) in Eq. (2) for $\psi_{y}$, we obtain the following dispersion relation in the case of the 


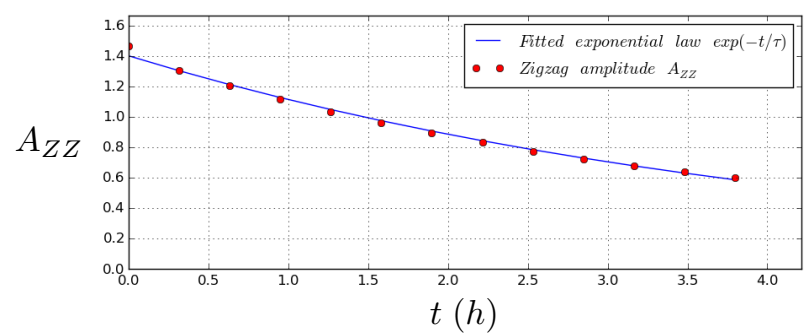

Figure 7: Evolution of $A_{Z Z}$ with time : relaxation of the zigzag amplitude over $3.8 \mathrm{~h}$, fitted by an exponential law $A_{Z Z} \sim e^{-t / \tau}$ (time constant $\tau_{Z Z}=4.37 h$ ).

zigzag instability :

$$
\Omega_{Z Z}=-D_{y x} K_{Z Z}^{2}
$$

We have confirmed the quadratic dependence of $\Omega_{Z Z}$ on $K_{Z Z}$ in (11) by means of additional simulations of zigzag instabilities of various wavelengths, thus supporting further the hypothesis of the diffusion equations (2) for the temporal evolution of $\psi_{x}$ and $\psi_{y}$.

Eq. (11) allows for the phase diffusion coefficient $D_{y x}$ to be numerically evaluated in our calculations, and the values obtained for zigzag instabilities of various wavelengths are within the range of $150 \mathrm{~nm}^{2} / \mathrm{s}$ to $350 \mathrm{~nm}^{2} / \mathrm{s}$. These values are close to that of the interdiffusion coefficient taken in our calculations $\left(D_{A l / N i}=217 \mathrm{~nm}^{2} / \mathrm{s}\right)$. Though the comparison of the interdiffusion coefficient $D_{A l / N i}$ with the phase diffusion coefficient $D_{y x}$ may seem premature, it appears to hold some significance since the alignment of precipitates during the zigzag relaxation can only occur by elasticity-induced diffusion of $\mathrm{Al}$ atoms, resulting in the simultaneous dissolution and growth of precipitates disposed at energetically-unfavorable positions in the pattern.

\section{Conclusions and Prospects}

The phase-based analysis has been shown to be both relevant and efficient as a means to topologically describe microstructure evolution during elasticity-induced coarsening. The response of a perfectly regular microstructure undergoing Eckhaus and zigzag instabilities has been investigated, and measured with $\psi_{x}$ and $\psi_{y}$. The system has revealed itself to be respectively unstable and stable against Eckhaus-type and zigzag-type distorsions of its regularity. The amplification of the Eckhaus instability leads to additional fluctuations of $\lambda_{x}$ which are traced by growing phase modes of various wavelengths, until precipitates coalesce. The zigzag instability relaxes at first, but the alignment process brings about the development of an Eckhaus instability whose growth becomes faster than the zigzag decay, eventually leading to precipitates coalescence before the complete relaxation of the initial instability.

In this work, we presented the perturbative analysis which we carried out on regular patterns with 1D topological defects. Aside from the latters, we also focused on 2D topological defects ("vacancy" and "dislocation" in the precipitates network, etc.) whose complex evolution may be quantitatively described using $\psi_{x}$ and $\psi_{y}$ as well. The transition from $2 \mathrm{D}$ to $3 \mathrm{D}$ topological analysis of microstructures will require the introduction of a third phase $\psi_{z}$ and of the corresponding equation for its dynamic. Therefore, three diffusion equations will be needed for the temporal evolution of $\psi_{x}, \psi_{y}$ and $\psi_{z}$, which, if taken at low-order, will each possess one Eckhaustype and two zigzag-type terms. The relevance of the phase-diffusion equations must be ascertained beforehand.

We intend to further develop this work by investigating the influence of the physical parameters of the system (i.e. temperature, misfit, volume fraction, elastic inhomogeneity, translational variants, external loading, etc.) and of the features of the topological defects (i.e. wavelength, amplitude, position in the pattern, interactions with other defects, etc.) on phases dynamics, in order to either confirm or overturn the relevance of phase equations for their temporal evolution.

In light of the results we have exposed in the present work, the phase-based analysis of microstructures appears promising, and seems to open new prospects for the modeling of microstructural evolution in these alloys at macroscale.

\section{References}

1. F. Larché, J.W. Cahn, "A linear theory of thermochemical equilibrium of solids under stress", Acta Metall., 21 (1973), 1051-1063.

2. F. Larché, J.W. Cahn, "A nonlinear theory of 
thermochemical equilibrium of solids under stress", Acta Metall., 26 (1978), 53-60.

3. F. Larché, J.W. Cahn, "Thermochemical equilibrium of multiphase solids under stress", Acta Metall., 26 (1978), 1579-1589.

4. W.C. Johnson, J.I. Alexander, "Interfacial conditions for thermomechanical equilibrium in two-phase crystals", J. Appl. Phys., 59 (1986), 2735-2746.

5. W.C. Johnson, P.W. Voorhees, "Phase equilibrium in two-phase coherent solids", Metall. Trans. A, 18A (1987), 1213-1228.

6. P. Caron, "High $\gamma$ ' solvus new generation nickelbased superalloys for single crystal turbine blade applications", Superalloys 2000, ed. T.M. Pollock et al. (Warrendale, PA, USA: TMS, 2000), 737-746.

7. W.C. Johnson, "Precipitate shape evolution under applied stress - Thermodynamics and kinetics", Metall. Trans. A, 18A (1987), 233-247.

8. A.C. Lund, P.W. Voorhees, "A quantitative assessment of the three-dimensional microstructure of a $\gamma$ - $\gamma^{\prime}$ alloy", Phil. Mag., 83 (2003), 1719-1733.

9. M.E. Thompson, C.S. Su, P.W. Voorhees, "The equilibrium shape of a misfitting precipitate", Acta Metall. Mater., 42 (1994), 2107-2122.

10. C.H. Su, P.W. Voorhees, "The dynamics of precipitate evolution in elastically stressed solids - I. Inverse coarsening", Acta Mater., 44 (1996), 1987-1999.

11. C.H. Su, P.W. Voorhees, "The dynamics of precipitate evolution in elastically stressed solids - II. Particle alignment", Acta Mater., 44 (1996), 2001-2016.

12. H. Yamauchi, D. De Fontaine, "Elastic interaction of defect clusters with arbitrary strain fields in an anisotropic continuum", Acta Metall., 27 (1979), 763-776.

13. W.C. Johnson, T.A. Abinandanan, P.W Voorhees, "The coarsening kinetics of two misfitting particles in an anisotropic crystal", Acta Metall. Mater., 38 (1990), 1349-1367.
14. T.A. Abinandanan, W.C. Johnson, "Coarsening of elastically interacting coherent particles - II. Simulations of preferential coarsening and particle migrations", Acta Metall. Mater., 41 (1993), 27-39.

15. A. Baldan, "Review - Progress in Ostwald ripening theories and their applications to nickel-base superalloys. Part I : Ostwald ripening theories", J. Mater. Sci., 37 (2002), 2171-2202.

16. H.J. Jou, P.H. Leo, J.S. Lowengrub, "Microstructural evolution in inhomogeneous elastic media", J. Comp. Phys., 131 (1997), 109-148.

17. K. Thornton, N. Akaiwa, P.W. Voorhees, "Largescale simulations of Ostwald ripening in elastically stressed solids : I. Development of microstructure", Acta Mater., 52 (2004), 1353-1364.

18. K. Thornton, N. Akaiwa, P.W. Voorhees, "Largescale simulations of Ostwald ripening in elastically stressed solids : II. Coarsening kinetics and particle size distribution", Acta Mater., 52 (2004), 1365-1378.

19. S.Y. Hu, L.Q. Chen, "A phase-field model for evolving microstructures with strong elastic inhomogeneity", Acta Mater., 49 (2001), 1879-1890.

20. M. Cottura, Y. Le Bouar, B. Appolaire, A. Finel, "Role of elastic inhomogeneity in the development of cuboidal microstructures in Ni-based superalloys", Acta. Mater., 94 (2015), 15-25.

21. L. Nguyen, R. Shi, Y. Wang, M. De Graef, "Quantification of rafting of $\gamma$ ' precipitates in Ni-based superalloys", Acta Mater., 103 (2016), 322-333.

22. M. Doi, T. Miyazaki, "Effect of elastic interaction energy on the distribution of coherent precipitate particles in nickel-base alloys", Superalloys 1992, ed. S.D. Antolovich et al. (Warrendale, PA, USA : TMS, 1992), 537-546.

23. M.C. Cross, P.C. Hohenberg, "Pattern formation outside of equilibrium", Rev. Mod. Phys., 65 (1993), 851-1112.

24. F.H. Busse, J.A. Whitehead, "Instabilities of convection rolls in a high Prandtl number fluid", J. Fluid Mech., 47 (1971), 305-320. 
25. Y. Pomeau, P. Manneville, "Stability and fluctuations of a spatially periodic convective flow", Le Journal de Physique - Lettres, 40 (1979), 609-612.

26. S. Akamatsu, M. Plapp, G. Faivre, A. Karma, "Overstability of Lamellar Eutectic Growth below the Minimum-Undercooling Spacing", Metall. and Mater. Trans., 35A (2004), 1815-1828.

27. G. Boussinot, Y. Le Bouar, A. Finel, "Phase-field simulations with inhomogeneous elasticity: Comparison with an atomic-scale method and application to superalloys", Acta Mater., 58 (2010), 4170-4181.

28. H. Sakaguchi, "Defect Creation by the Eckhaus Instability", Prog. Theor. Phys., 85 (1991), 927-932.

29. J. Millán-Rodríguez, M. Bestehorn, C. PérezGarcía, R. Friedrich, M. Neufeld, "Defect Motion in Rotating Fluids", Phys. Rev. Lett., 74 (1995), 530533. 